\title{
Vandetanib-induced inhibition of neuroblastoma cell migration and invasion is associated with downregulation of the SDF-1/CXCR4 axis and matrix metalloproteinase 14
}

\author{
XIONGHUI DING $^{1}$, LI XIANG ${ }^{2}$, NING WANG $^{1}$, ZHANBO ZHAO $^{1}$, XIN JIN $^{3}$, YANHUI SUN ${ }^{4}$, \\ WENJUAN DUAN ${ }^{1}$, SHIQI WANG ${ }^{1}$ and XIANQING JIN ${ }^{1,2}$ \\ ${ }^{1}$ Ministry of Education Key Laboratory of Child Development and Disorders; ${ }^{2}$ Department of Neonatal \\ Gastrointestinal Surgery; ${ }^{3}$ Key Laboratory of Pediatrics in Chongqing; ${ }^{4}$ Chongqing International Science \\ and Technology Cooperation Center for Child Development and Disorders, Children's Hospital \\ of Chongqing Medical University, Yuzhong, Chongqing 400014, P.R. China
}

Received October 29, 2013; Accepted December 5, 2013

DOI: 10.3892/or.2013.2963

\begin{abstract}
Rearranged during transfection (RET) is widely expressed in neuroblastoma (NB) and partly contributes to high metastatic potential and survival of NB. The aim of the present study was to investigate whether vandetanib (a RET inhibitor) inhibits proliferation, migration and invasion of NB cells in vitro. The effects of vandetanib on the proliferation, apoptosis and cell cycle and on RET phosphorylation of SK-N-SH and SH-SY5Y cells were evaluated in vitro. The migration and invasion potential of vandetanib-treated NB cells were analyzed using Transwell cell migration and invasion assays, respectively. qPCR, western blotting and immunofluorescence were used to detect mRNA and protein levels in NB cells treated with vandetanib. Our data demonstrated that vandetanib inhibits the proliferation of SK-N-SH and SH-SY5Y cells and that this inhibition is mediated by the induction of G1 phase cell cycle arrest at lower concentrations and by apoptosis at higher concentrations. In the presence of vandetanib, the migration and invasion of two NB cell lines were markedly decreased compared with the control group $(\mathrm{p}<0.01)$. In addition, our data showed that the levels of C-X-C chemokine receptor type 4 (CXCR4) and matrix metalloproteinase 14 (MMP14) mRNA expression in NB cell lines treated with vandetanib were significantly lower than those in the cells that were treated with vehicle $(\mathrm{p}<0.01)$ and similar results were obtained for protein levels as determined by western blotting and immunofluorescence analysis. Vandetanib may inhibit the proliferation, migration and invasion of NB cells in vitro.
\end{abstract}

Correspondence to: Professor Xianqing Jin, Department of Neonatal Gastrointestinal Surgery, Children's Hospital of Chongqing Medical University, 136 Zhongshan 2nd Road, Yuzhong, Chongqing 400014, P.R. China

E-mail: etzhl@163.com

Key words: neuroblastoma, vandetanib, tumor metastasis, chemokine receptor type 4, matrix metalloproteinase 14
The potential mechanisms for the inhibition of NB migration and invasion by vandetanib may partly be attributed to the ability of vandetanib to suppress the expression of CXCR4 and MMP14 in human NB cells.

\section{Introduction}

Neuroblastoma (NB) is one of the most frequently occurring pediatric solid tumors and is derived from ganglionic lineage precursors of the sympathetic nervous system. Patients with high-risk NB have very poor outcomes with 5-year disease-free survival rates between 25 and 35\% (1,2). Distant metastasis, particularly bone metastasis, is one of the leading causes of mortality in patients with NB.

Previous studies have shown that most NB cell lines express rearranged during transfection (RET), and overexpression or activation of RET alters cell adhesion and enhances metastatic behavior and non-adherent proliferation of NB cells, which may contribute to NB metastasis $(3,4)$. The RET proto-oncogene, which encodes a receptor tyrosine kinase known to be expressed on the surface of neuronal and neural crest-derived cells, is critical for development of the enteric nervous system (ENS) and kidneys. However, gain-of-function mutation of RET is associated with the development of various types of human cancer, including medullary thyroid carcinoma, multiple endocrine neoplasias type $2 \mathrm{~A}$ and $2 \mathrm{~B}$, pheochromocytoma and NB (5). During kidney development, Lu et al found that the ETS transcription factors Etv4 and Etv5 are positively regulated by RET/GDNF signaling in the ureteric bud tips, and they identified several genes whose expression in the ureteric bud depends on Etv4 and Etv5, including C-X-C chemokine receptor type 4 (CXCR4), Myb, Met and Mmp14 (6). CXCR4 is an $\alpha$-chemokine receptor specific for stromal-derivedfactor-1 (SDF-1, also called CXCL12), and both are involved in metastasis in various malignancies, such as NB, prostate and breast cancer (7-9). Matrix metalloproteinases (MMPs), including MMP14, are capable of degrading several types of extracellular matrix proteins. MMPs are also thought to play a major role in cell invasion in tumor metastasis $(10,11)$. 
Vandetanib (ZD6474) is an orally active small molecule tyrosine kinase inhibitor with activity against the RET tyrosine kinase, VEGF receptor 2 (VEGFR2), VEGF receptor 3 (VEGFR3) and epidermal growth factor receptor (EGFR) $(12,13)$. Current studies have shown that vandetanib is effective against NB tumor cells and reduces NB xenograft tumor growth rates, whereas bevacizumab and erlotinib, inhibitors of the VEGFR and EGFR pathways, respectively, do not hinder tumor growth $(14,15)$. Moreover, the results of the present study showed that SK-N-SH cells lack EGFR and VEGFR2, and phosphorylated RET was consistently expressed at high levels in NB cell lines including SK-N-SH and SH-SY5Y, with undetectable levels of phosphorylated EGFR and VEGFR2. These results indicate antitumor activity by vandetanib should be mainly mediated by inhibition of phosphorylated RET.

The aim of the present study was to investigate the activity and potential mechanisms of vandetanib against the migration and invasion of NB in vitro. Due to the evidence of the roles of RET signaling in NB pathogenesis and CXCR4 and MMP14 being downstream of RET signaling, we hypothesized that vandetanib-induced inhibition of NB migration and invasion may be associated with downregulation of the SDF-1/CXCR4 axis and MMP14.

\section{Materials and methods}

Reagents. Vandetanib was purchased from LC Laboratories (Woburn, MA, USA). A stock solution $(50 \mathrm{mM})$ was produced in dimethyl sulfoxide (DMSO; Sigma-Aldrich) and stored at $-20^{\circ} \mathrm{C}$. AMD3100 (Sigma; cat: $155148-31-5$ ) was soluble in water at $22 \mathrm{mg} / \mathrm{ml}$ and stored at $-20^{\circ} \mathrm{C}$. Recombinant human GDNF (cat: 450-10) and recombinant human SDF-1 $\alpha / C X C L 12$ (cat: 300-28A) (both from PeproTech) were diluted in buffer containing $0.1 \%$ bovine serum albumin (BSA) and stored in working aliquots at $-20^{\circ} \mathrm{C}$.

Cell lines and culture conditions. SK-N-SH cells were maintained in Dulbecco's modified Eagle's medium (DMEM) supplemented with $10 \%$ fetal bovine serum (FBS) (Gibco), $100 \mathrm{U} / \mathrm{ml}$ penicillin and $100 \mu \mathrm{g} / \mathrm{ml}$ streptomycin. SH-SY5Y cells were maintained in DMEM-F12 supplemented with $10 \%$ FBS, $100 \mathrm{U} / \mathrm{ml}$ penicillin and $100 \mu \mathrm{g} / \mathrm{ml}$ streptomycin. The two NB cell lines were maintained at $37^{\circ} \mathrm{C}$ in a humidified atmosphere with $5 \%$ carbon dioxide.

In vitro proliferation, apoptosis and cell cycle assay. A cell proliferation assay was performed with 3-(4,5-dimethylthiazol-2-yl)-2,5-diphenyltetrazolium bromide (MTT) (Sigma; cat: M2128). Before determining proliferation, SK-N-SH or SH-SY5Y cells were seeded into 96-well plates in quintuplicate and allowed to adhere in complete medium overnight. The medium was removed and $100 \mu \mathrm{l}$ of fresh medium containing the control vehicle or vandetanib $(0.625$, $1.25,2.5,5,10$ and $20 \mu \mathrm{M}$ ) was added. After $48 \mathrm{~h}, 15 \mu \mathrm{l}$ of MTT was added to each well. After $4 \mathrm{~h}$ of incubation, the medium was removed, $200 \mu \mathrm{l}$ of DMSO was added, and the optical density was measured at $570 \mathrm{~nm}$ in a multi-well plate reader (Thermo Scientific). The background absorbance of the medium in the absence of cells was subtracted, and the results were expressed as a percentage of the control, which was defined as $100 \%$.

Apoptosis was measured using the Annexin V-FITC Apoptosis Detection kit (KeyGen Biotech, China; cat: KG107) according to the manufacturer's instructions. Apoptotic cells were differentiated from viable or necrotic cells by the combined application of Annexin V-FITC and propidium iodide (PI). Briefly, cells were incubated in the presence of vehicle (DMSO), vandetanib $(5 \mu \mathrm{M})$, vandetanib $(10 \mu \mathrm{M})$ or vandetanib $(20 \mu \mathrm{M})$ for $48 \mathrm{~h}$. The samples were harvested and washed with $4^{\circ} \mathrm{C}$ PBS. A total of $1 \times 10^{6}$ cells were resuspended in $500 \mu 1$ of $1 \mathrm{X}$ binding buffer, incubated with $5 \mu \mathrm{l}$ of Annexin V-FITC and $5 \mu \mathrm{l}$ of PI solution and finally incubated for $15 \mathrm{~min}$ at room temperature in the dark. The fluorescence of the cells was determined immediately and analyzed with a flow cytometry (FCM) analyzer (BD Biosciences, USA). This assay was performed in triplicate.

The cell cycle assays were analyzed with an FCM analyzer. Both floating and adherent cells were harvested after incubating in the presence of vehicle (DMSO), vandetanib $(5 \mu \mathrm{M})$, vandetanib $(10 \mu \mathrm{M})$ or vandetanib $(20 \mu \mathrm{M})$ for $48 \mathrm{~h}$, washing with $4^{\circ} \mathrm{C}$ PBS and fixing overnight with ice-cold $70 \%$ ethanol at $4^{\circ} \mathrm{C}$. Cells were centrifuged to obtain a pellet, resuspended in $500 \mu \mathrm{l}$ of hypotonic buffer $(0.5 \%$ Triton X-100 and $0.5 \mathrm{~g} / \mathrm{ml}$ RNase A in ice-cold PBS) and incubated for $30 \mathrm{~min}$ at $37^{\circ} \mathrm{C}$. Then, $1 \mathrm{ml}$ of PI solution $(50 \mu \mathrm{g} / \mathrm{ml})$ was added and incubated for $30 \mathrm{~min}$ at $4^{\circ} \mathrm{C}$ to form a PI-DNA complex. The percentage of the cell population in each phase of the cell cycle was measured using FACStar flow cytometer, and the results were analyzed using CellQuest software (Becton-Dickinson and Company, Franklin Lakes, NJ, USA).

Detection of RET phosphorylation. NB cells were treated with vehicle (DMSO), vandetanib (1,5 and $10 \mu \mathrm{M}$, respectively) for $48 \mathrm{~h}$, rinsed twice with ice-cold PBS and lysed in protein lysis buffer (0.5\% Nonidet P-40, $10 \mathrm{mM}$ Tris- $\mathrm{HCl} \mathrm{pH} 7.4,150 \mathrm{mM}$ $\mathrm{NaCl}, 1 \mathrm{mM}$ EDTA and $1 \mathrm{mM} \mathrm{Na} \mathrm{VO}_{4}$ ) supplemented with protease inhibitors, calcineurin inhibitors and $1 \mathrm{mM}$ phenylmethylsulfonyl fluoride (PMSF). A total of $30 \mu \mathrm{g}$ of protein was separated by 6-12\% SDS-polyacrylamide gel electrophoresis (SDS-PAGE) and transferred to polyvinylidene fluoride (PVDF) membranes (Millipore, Billerica, MA, USA). The membranes were blocked for $1 \mathrm{~h}$ with 5\% BSA (Bovine Serum Albumin Fraction V; Roche, 10735078001) in TBS-T [10 mM Tris-HCl pH 7.5, $100 \mathrm{mM} \mathrm{NaCl}$ and $0.1 \%$ (w/v) Tween-20]. The membranes were then incubated overnight at $4^{\circ} \mathrm{C}$ with primary antibodies against $\beta$-actin, p-EGFR (cat: 3777s), p-VEGFR2 (cat: 2478s) (both from Cell Signaling Technology, Beverly, MA, USA) or p-RET (Santa Cruz Biotechnology, Santa Cruz, CA, USA; cat: sc-20252-R). The membranes were rinsed three times with TBS-T and incubated with an HRP-conjugated goat anti-rabbit or goat anti-mouse $\operatorname{IgG}(\mathrm{H}+\mathrm{L})$ secondary antibody. The blots were visualized using an ECL kit (Tiangen Biotech Co., Ltd., Beijing, China).

Transwell cell migration assay. The in vitro migration of vandetanib-treated NB cells was assessed using a Transwell chemotaxis system. To analyze the role of the SDF-1/CXCR4 axis in the migration of vandetanib-treated NB cells, AMD3100, a highly specific chemokine receptor CXCR4 antagonist, 
served as a control. Non-coated culture plate well inserts (Millipore) with $8-\mu \mathrm{m}$ pore size polycarbonate membranes (upper chambers) were inserted into the 24-well plates (lower chambers), and DMEM or DMEM-F12 supplemented with $10 \%$ FBS containing $100 \mathrm{ng} / \mathrm{ml} \mathrm{hSDF}-1 \mathrm{a}$ was added to each lower chamber. Before migration, NB cells were cultured for $72 \mathrm{~h}$ in the presence of vehicle (DMSO), vandetanib $(5 \mu \mathrm{M})$, vandetanib $(10 \mu \mathrm{M})$, AMD3100 $(10 \mu \mathrm{M})$, vandetanib $(5 \mu \mathrm{M})$ or vandetanib plus AMD3100. Then, the floating apoptotic cells were rinsed away, the cells were harvested and $100 \mu \mathrm{l}$ of cells at a concentration of $3 \times 10^{5}$ cells $/ \mathrm{ml}$ was seeded into the upper chamber of each Transwell and allowed to migrate for $24 \mathrm{~h}$ at $37^{\circ} \mathrm{C}$ in $5 \% \mathrm{CO}_{2}$. After incubation, $\mathrm{NB}$ cells on the upper surface of the insert membrane were gently removed with cotton tips. Cells on the lower surface of the membrane were fixed with $100 \%$ methanol for $15 \mathrm{~min}$ and then stained with DAPI (DAPI-dilactose; Sigma) for $15 \mathrm{~min}$. Each treatment was independently repeated three or four times in duplicate. The total number of cells in four random fields of each membrane was counted at 10x10 magnification with a fluorescence microscope (Nikon Instruments Inc., Japan).

Transwell invasion assay. A Transwell invasion assay was performed using a BD BioCoat Matrigel ${ }^{\mathrm{TM}}$ 24-well invasion chamber with $8-\mu \mathrm{m}$ pores (BD Biosciences). The Matrigel BioCoat inserts were initially rehydrated by adding serumpoor medium in both the upper and lower chambers and incubated in a tissue culture incubator for $2 \mathrm{~h}$. After removal of the rehydrating medium, DMEM or DMEM-F12 supplemented with $10 \%$ FBS containing $100 \mathrm{ng} / \mathrm{ml}$ hSDF-1a was added to each lower chamber. The invasion assays were designed in the same five configurations as the Transwell cell migration assays. After being treated with vehicle (DMSO), vandetanib $(5 \mu \mathrm{M})$, vandetanib $(10 \mu \mathrm{M})$, AMD3100 $(10 \mu \mathrm{M})$, vandetanib $(5 \mu \mathrm{M})$ or vandetanib plus AMD3100 for $72 \mathrm{~h}$, the floating apoptotic cells were rinsed away, the cells were harvested and $100 \mu \mathrm{l}$ of cells at a concentration of $3 \times 10^{5}$ cells $/ \mathrm{ml}$ was seeded into the upper chamber insert with the Matrigel coated membrane. The plates were then incubated at $37^{\circ} \mathrm{C}$ in $5 \% \mathrm{CO}_{2}$ for $48 \mathrm{~h}$. After incubation, noninvading cells and the Matrigel matrix on the upper surface of the membrane were gently removed with cotton tips. The cells on the lower surface of the membrane were fixed with $100 \%$ methanol for $15 \mathrm{~min}$ and then stained with DAPI (DAPI-dilactose) for $15 \mathrm{~min}$. Each treatment was independently repeated three times in duplicate. The total number of cells in four random fields of each membrane was counted at 10x20 magnification with a fluorescence microscope (Nikon Instruments Inc.).

RT-qPCR analysis. Briefly, total RNA from NB cells was isolated with TRIzol reagent (Invitrogen; cat: 15596-026) and reverse transcribed with the PrimeScript ${ }^{\mathrm{TM}} \mathrm{RT}$ reagent kit (Takara; cat: DRR037A). Quantitative real-time PCR (qPCR) was performed in triplicate in $10-\mu 1$ reactions using the CFX96 Real-Time System (Bio-Rad, USA). The reaction solution contained $20 \mathrm{ng}$ of reverse transcribed total RNA, $0.4 \mu \mathrm{M}$ of each paired primer and $5 \mu$ of SYBR Premix Ex Taq ${ }^{\mathrm{TM}}$ II (Takara; cat: DRR820A). Amplification was performed using the following conditions: $95^{\circ} \mathrm{C}$ for $30 \mathrm{sec}$ followed by 40 cycles of $95^{\circ} \mathrm{C}$ for $10 \mathrm{sec}$ and $60^{\circ} \mathrm{C}$ for $30 \mathrm{sec}$. The specificity of each amplified qPCR product was examined by dissociation curve analysis. The temperature range to detect the melting temperature of the PCR product was set from 65 to $95^{\circ} \mathrm{C}$. The relative expression was calculated using the comparative $\mathrm{Ct}$ (threshold cycle) method with the arithmetic formula $2^{-\Delta \Delta \mathrm{Ct}}$. A standard curve of serially diluted PCR products of cDNA was used to correlate changes in $\Delta \mathrm{Ct}$ with fold changes in the mRNA levels of each gene. Amplification of the housekeeping gene $\beta$-actin was measured for each sample as an internal control for sample loading and normalization. The results are representative of at least three independent experiments. Primers used for PCR analysis were: RET forward, 5'-TTTG CCTGGCAGATCTCACA-3' and reverse, 5'-GATGTTTCTG GCTGCCAAGTC-3'; CXCR4 forward, 5'-TTCTACCCCAAT GACTTGTG-3' and reverse, 5'-ATGTAGTAAGGCAGCCA ACA-3'; MMP14 forward, 5'-TGCCTGCGTCCATCAACA-3' and reverse, 5'-ATCTTGTCGGTAGGCAGC-3'; $\beta$-actin forward, 5'-AAGATGACCCAGATCATGTTTGAGACC-3' and reverse, 5'-GCCAGGTCCAGACGCAGGAT-3'.

Western blotting. Total cell protein extracts were obtained as described above from NB cells, which were cultured in the presence of vehicle (DMSO), vandetanib $(5 \mu \mathrm{M})$ for $48 \mathrm{~h}$ or vandetanib $(5 \mu \mathrm{M})$ for $72 \mathrm{~h}$. A total of $30 \mu \mathrm{g}$ of protein was separated by $6-12 \%$ SDS-PAGE and transferred to PVDF membranes. The membranes were blocked for $1 \mathrm{~h}$ with $5 \%$ BSA in TBS-T, then incubated overnight at $4^{\circ} \mathrm{C}$ with primary antibodies against $\beta$-actin, p-RET, RET (Santa Cruz Biotechnology, Inc.; cat: sc-167), CXCR4 (Abcam; cat: ab2074) or MMP14 (Santa Cruz Biotechnology, Inc.; cat: sc-30074). The membranes were rinsed three times with TBS-T and incubated with an HRP-conjugated goat anti-rabbit or goat anti-mouse IgG $(\mathrm{H}+\mathrm{L})$ secondary antibody. The blots were visualized using an ECL kit (Tiangen Biotech Co., Ltd.).

Immunofluorescence. For the immunofluorescence staining, $1 \times 10^{5}$ cells were cultured on glass coverslips in 24-well plates overnight, and then treated with $5 \mu \mathrm{M}$ vandetanib or vehicle (DMSO) for $48 \mathrm{~h}$. All the samples were washed twice with PBS and fixed with ice-cold methanol for $15 \mathrm{~min}$. The samples were blocked with $5 \%$ BSA for $1 \mathrm{~h}$ at room temperature, incubated overnight at $4^{\circ} \mathrm{C}$ with primary antibody against p-RET, and then incubated with TRITC-conjugated secondary antibody in the dark for $1 \mathrm{~h}$ at room temperature. All the subsequent procedures were performed in the dark. The samples were incubated overnight at $4^{\circ} \mathrm{C}$ with primary antibody against CXCR4, followed by an FITC-conjugated secondary antibody for $1 \mathrm{~h}$ at room temperature and counterstaining with DAPI for $15 \mathrm{~min}$ at room temperature. The coverslips were sealed to microscope slides with DABCO, and the cells were imaged with a fluorescence microscope (Nikon Instruments Inc.).

Statistical analysis. Significant differences between groups were determined for apoptosis, cell cycle, migration, invasion and mRNA expression studies using an ANOVA test with multiple comparisons analyzed by SPSS (version 17.0) software. The results are presented as the means \pm SD. The minimal level of significance was set to $\mathrm{p}<0.05$. 


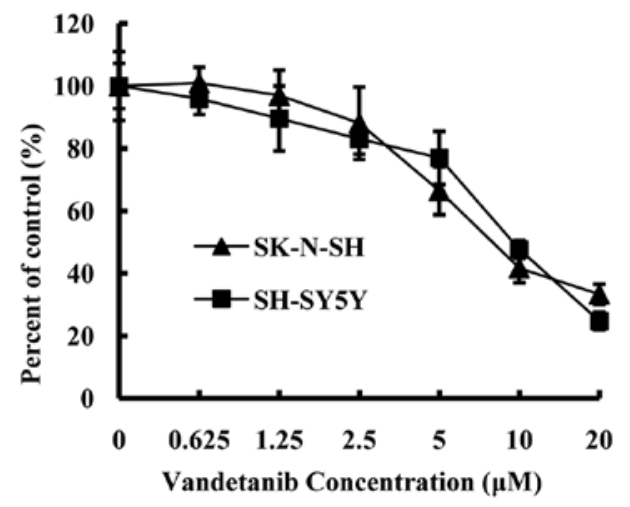

Figure 1. SK-N-SH and SH-SY5Y cells are sensitive to vandetanib. Cells were treated with vandetanib for $48 \mathrm{~h}$ and the $\mathrm{IC}_{50}$ value of vandetanib in SK-N-SH and SH-SY5Y cells was $\sim 10 \mu \mathrm{M}$. The results are expressed as a percentage of the control, which was defined as $100 \%$, shown as the means \pm SD of three independent experiments.

\section{Results}

Vandetanib inhibits the proliferation of human NB cell lines. To determine the $\mathrm{IC}_{50}$ value of vandetanib on $\mathrm{NB}$ cells in vitro, we treated SK-N-SH and SH-SY5Y cells with the control vehicle or vandetanib $(0.625,1.25,2.5,5,10$ and $20 \mu \mathrm{M})$ for $48 \mathrm{~h}$. We then evaluated the antiproliferative effect with an MTT assay. In the present study, the $\mathrm{IC}_{50}$ value of vandetanib on SK-N-SH and SH-SY5Y cells was $\sim 10 \mu \mathrm{M}$ (Fig. 1), which is consistent with previous studies $(14,15)$.
Vandetanib induces apoptosis in human NB cell lines. Based on the results of the MTT assay, vandetanib concentrations of 5, 10 and $20 \mu \mathrm{M}$, which inhibited the growth of the two cell lines by $\sim 25-75 \%$ over $48 \mathrm{~h}$, were used for apoptosis and cell cycle assay. To elucidate the mechanism by which vandetanib exerts its antiproliferative effect on NB cell lines, an Annexin V/PI binding assay was performed. Our data revealed an increased percentage of early apoptotic cells (Annexin $\mathrm{V}^{+} / \mathrm{PI}^{-}$) after treatment of SK-N-SH cells with $10 \mu \mathrm{M}$ vandetanib for $48 \mathrm{~h}(\mathrm{p}<0.05)$, and the percentage of early apoptotic cells increased significantly after increasing the vandetanib concentration to $20 \mu \mathrm{M}(\mathrm{p}<0.01)$. Vandetanib treatment of SK-N-SH cells also induced an increase in the fraction of late apoptotic/necrotic cells (Annexin $\mathrm{V}^{+} / \mathrm{PI}^{+}$) at concentrations of $10 \mu \mathrm{M}(\mathrm{p}<0.05)$ and $20 \mu \mathrm{M}(\mathrm{p}<0.01)$. There was no significant difference in the percentage of early apoptotic cells between the control group and the $5 \mu \mathrm{M}$ vandetanib group ( $\mathrm{p}>0.05$ ). Similar results were obtained with SH-SY5Y cells (Fig. 2).

Vandetanib induces $G 1$ phase cell cycle arrest in human NB cell lines. To investigate whether the antiproliferative effect of vandetanib on the NB cell lines was partly mediated via specific cell cycle arrest, we examined the cell cycle phase distribution of cells treated with vandetanib by flow cytometric analysis. Our data showed that vandetanib treatment caused a significant accumulation of cells in the G1 phase in the two cell lines (Fig. 3). The percentage of SK-N-SH cells in the $\mathrm{G} 1$ phase after treatment with 5 or $10 \mu \mathrm{M}$ vandetanib
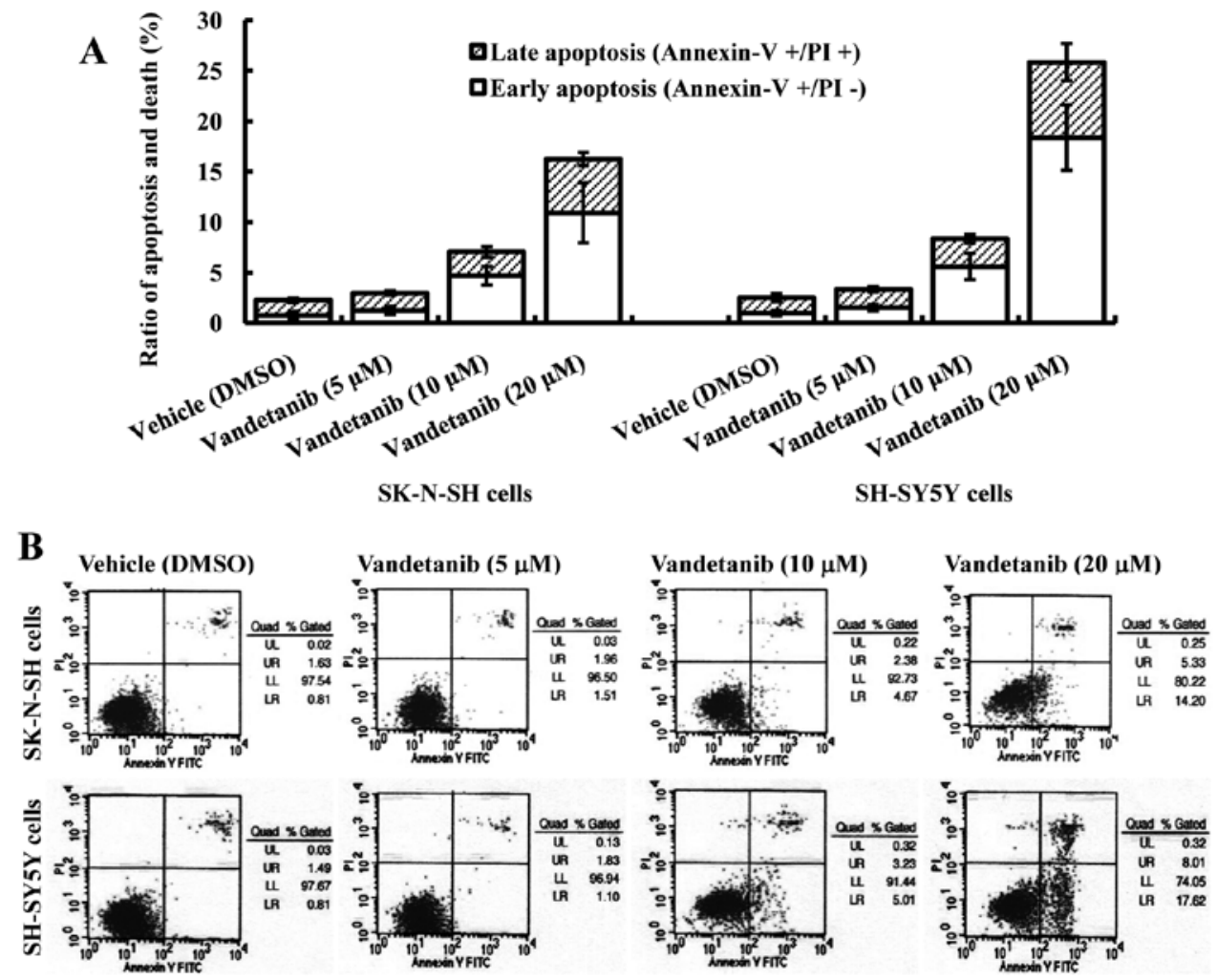

Figure 2. Induction of apoptosis and cell cycle arrest in human neuroblastoma cells by vandetanib in vitro. (A) Annexin V/propidium iodide (PI) binding assay analysis of apoptosis in vandetanib-treated SK-N-SH and SH-SY5Y cell lines. The percentages of early apoptosis (Annexin $\mathrm{V}^{+} / \mathrm{PI}$, LR) and late apoptosis (Annexin $\mathrm{V}^{+} / \mathrm{PI}^{+}$, UR) from these experiments are given. The results are expressed as the means \pm SD of three independent experiments. (B) Representative images of the respective treatments in the Annexin V/PI binding assay. 

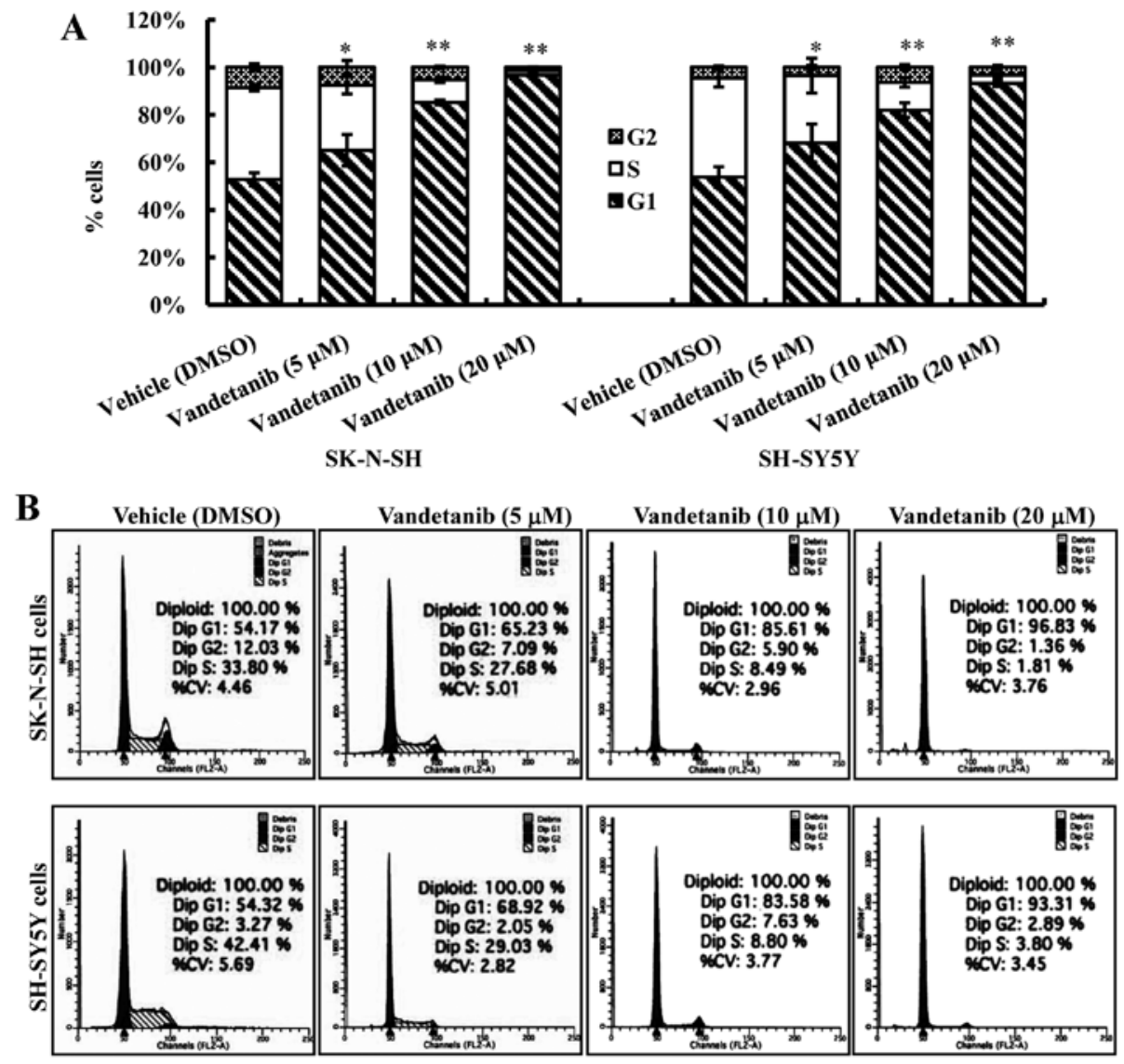

Figure 3. Vandetanib treatment causes significant accumulation of cells in the G1 phase in human neuroblastoma cell lines. (A) Cell cycle distribution of the two cell lines was determined by flow cytometric analysis. The total population of cells in each phase of the cell cycle is shown as the means \pm SD of three independent experiments. Statistically significant differences in the percentage of cells in the G1 phase relative to the control group were determined using a one-way ANOVA; ${ }^{*} \mathrm{p}<0.05,{ }^{* *} \mathrm{p}<0.01$ vs. control. (B) Representative flow cytometry analysis of the cell cycle distribution of the SK-N-SH and SH-SY5Y cell lines, which were released to induce G1 phase cell cycle arrest by vandetanib.
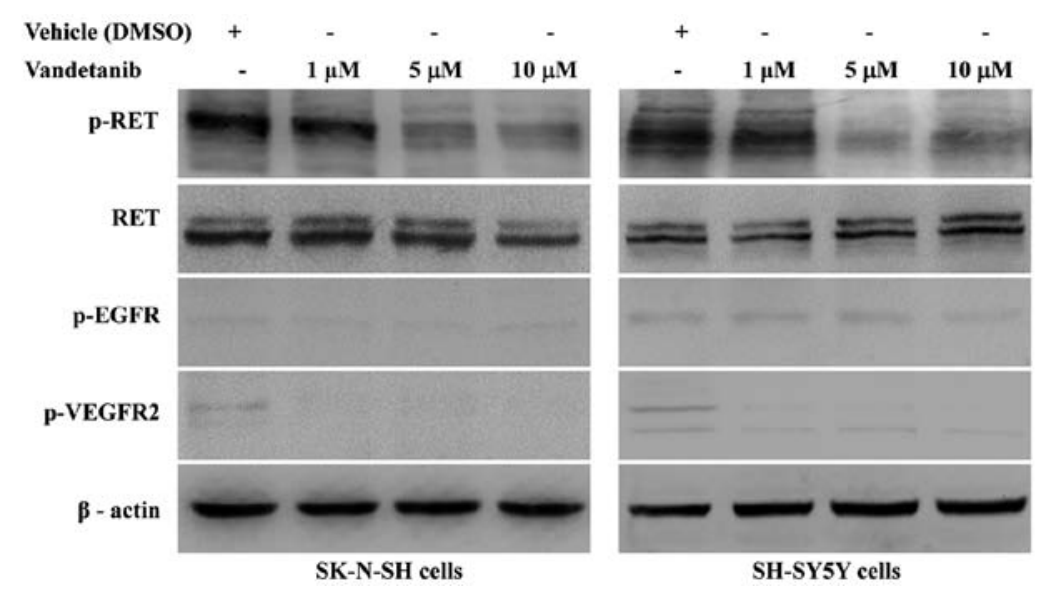

Figure 4. In vitro inhibition of RET phosphorylation by vandetanib. Phosphorylation of RET was markedly inhibited in cells treated with vandetanib for $48 \mathrm{~h}$ at concentrations of 5 or $10 \mu \mathrm{M}$. Extremely low levels of the phosphorylated EGFR and VEGFR2 were detectable in cells in the presence or absence of vandetanib.

$(65.10 \pm 6.58 \%, 85.25 \pm 0.99 \%)$ increased markedly compared with the cells treated with vehicle $(52.80 \pm 2.77 \%)(\mathrm{p}<0.01)$. The percentage of G1 phase cells continued to increase in SK-N-SH cells treated with $20 \mu \mathrm{M}$ vandetanib $(96.23 \pm 0.41 \%)(\mathrm{p}<0.01)$. Similar results were obtained in SH-SY5Y cells treated with vandetanib.
Vandetanib inhibits RET phosphorylation in human NB cell lines. To evaluate the inhibitory effect of vandetanib on RET activation, SK-N-SH and SH-SY5Y cells were treated with vandetanib $(1,5$ or $10 \mu \mathrm{M})$ for $48 \mathrm{~h}$. Whole-cell lysates from the two NB cell lines were evaluated by western blot analysis. As shown in Fig. 4, phosphorylated RET was detected in the 


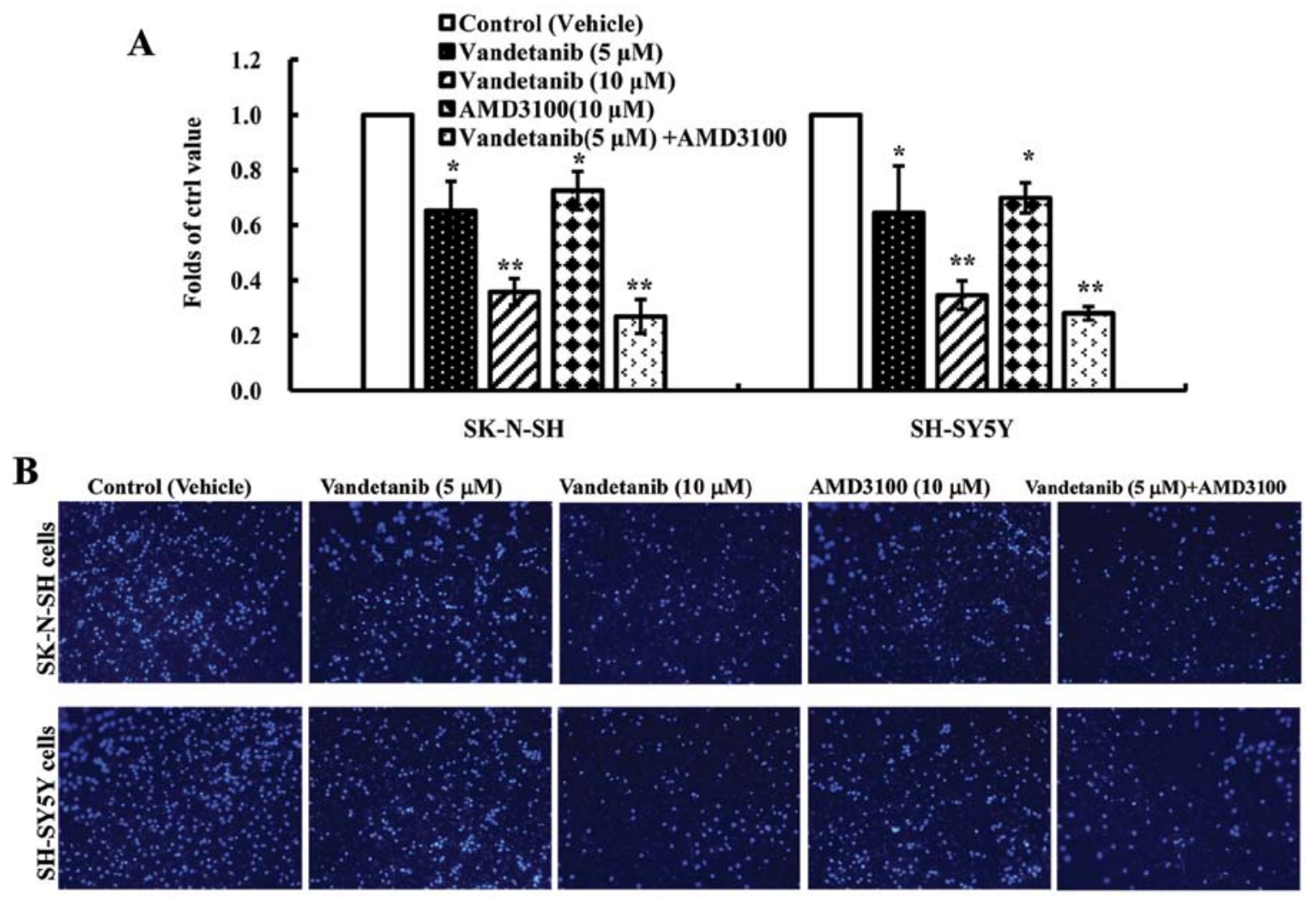

Figure 5. Transwell migration assay of the neuroblastoma cell lines SK-N-SH and SH-SY5Y. (A) The migration efficiency was illustrated as the fold increase or decrease in migrated cells over the control. Cells from each sample were counted in four random fields at $10 x 10$ magnification. The results are expressed as the relative fold-change compared with the corresponding control value (means $\pm \mathrm{SD}, \mathrm{n}=3$ or $n=5$ ); ${ }^{*} \mathrm{p}<0.05$, ${ }^{* *} \mathrm{p}<0.01$ vs. control. (B) Representative images of migrated cells treated with DMSO, vandetanib (5 and $10 \mu \mathrm{M}$, respectively), AMD3100, vandetanib (5 $\mu \mathrm{M})$ plus AMD3100.
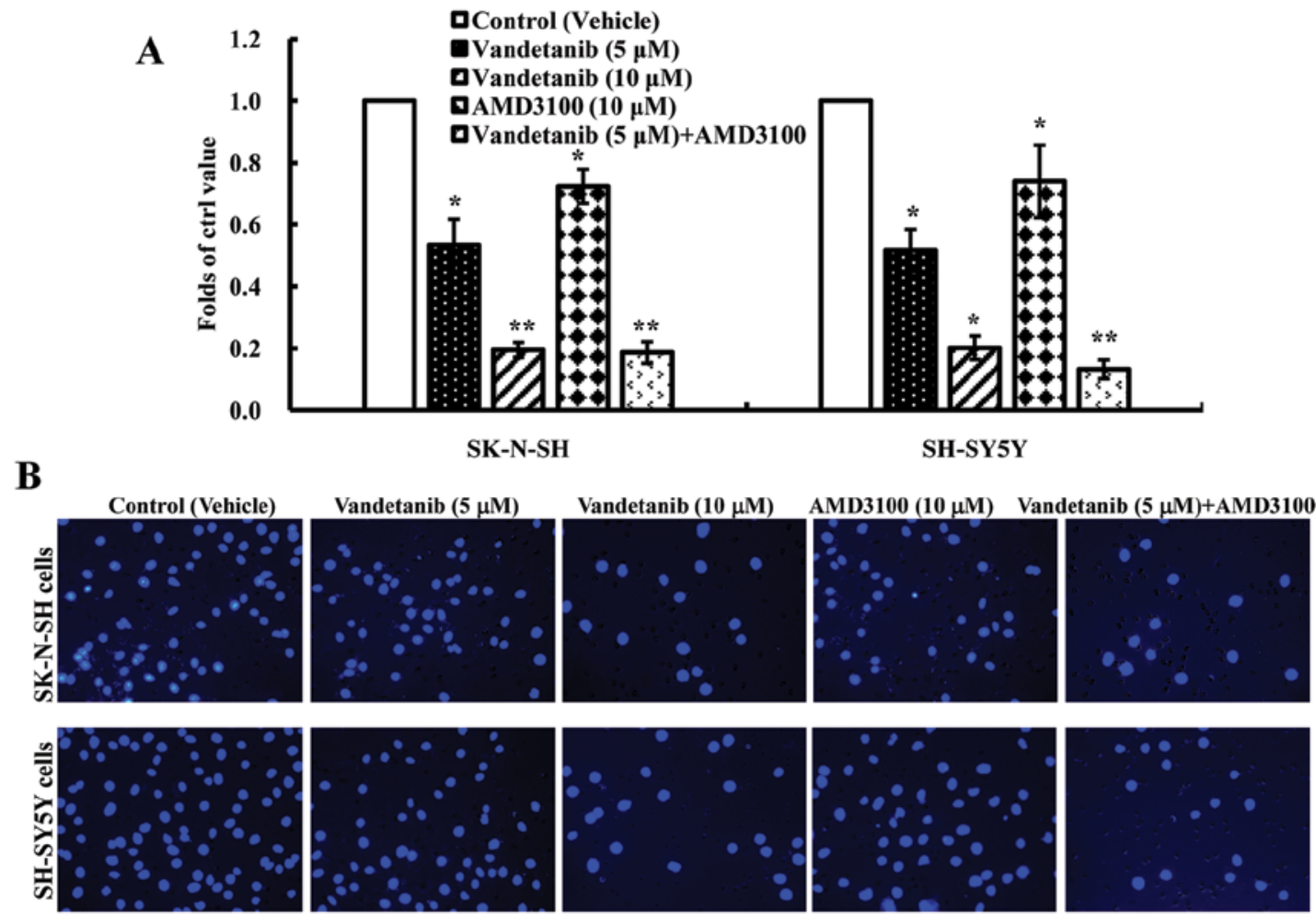

Figure 6. Transwell invasion assay of the neuroblastoma cell lines SK-N-SH and SH-SY5Y. (A) Cells from each sample were counted in four random fields at $10 \times 20$ magnification. The results are expressed as the relative fold-change compared with the corresponding control value $(m e a n s \pm S D, n=3$ or $n=4) ;{ }^{*} p<0.05$, ${ }^{* *}$ p $<0.01$ vs. control. (B) Representative images of cells treated with DMSO, vandetanib, AMD3100, vandetanib (5 $\mu$ M) plus AMD3100 that travelled through the Matrigel BioCoat micropore membrane. 


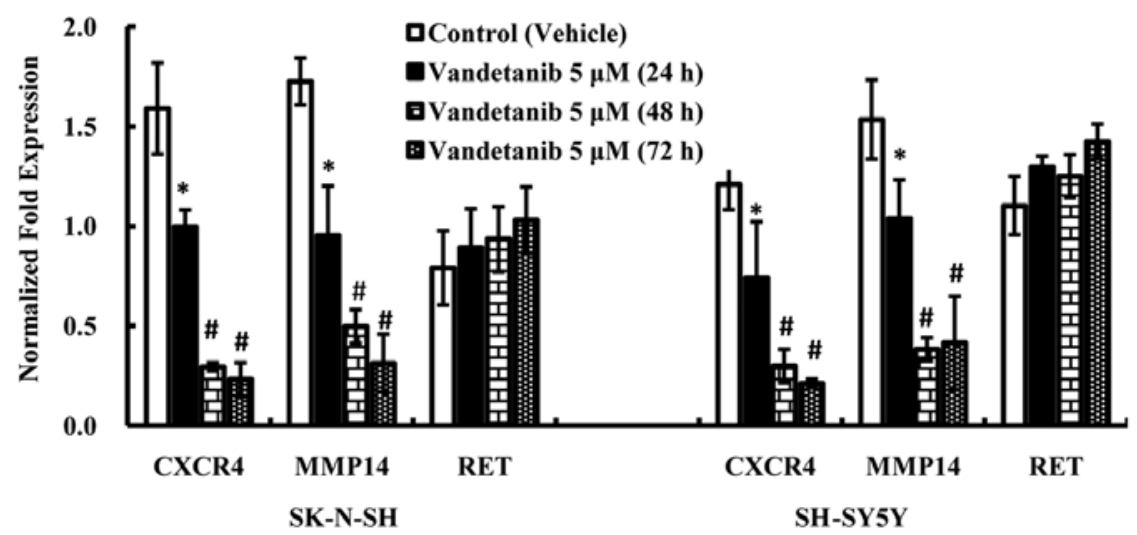

Figure 7. Vandetanib suppresses the expression of CXCR4 and MMP14 mRNA in human neuroblastoma cell lines. The transcript levels of CXCR4, MMP14 and RET mRNA were analyzed by qPCR and $\beta$-actin was included as a loading control. CXCR4 and MMP14 expression was significantly downregulated by vandetanib. The results are expressed as the means $\pm \mathrm{SD}$ from five separate experiments; ${ }^{*} \mathrm{p}<0.05,{ }^{\#} \mathrm{p}<0.01 \mathrm{vs}$. control. CXCR4, C-X-C chemokine receptor type 4; MMP14, matrix metalloproteinase 14.

two cell lines treated with vehicle (DMSO), and the protein level was markedly reduced in cells treated with $5 \mu \mathrm{M}$ vandetanib. There were no significant differences in phosphorylated RET levels in cells treated with 5 and $10 \mu \mathrm{M}$ vandetanib. However, extremely low levels of the phosphorylated EGFR and VEGFR2 were detectable in cells treated with vehicle, with minimal to no levels detectable in cells treated with vandetanib.

Vandetanib inhibits human NB cell migration in vitro. To investigate the inhibitory effect of vandetanib on the migration of NB cells in vitro, the migration efficiency of NB cells in response to serum and SDF-1 $\alpha$ was tested using a Transwell migration system. The migration efficiency was calculated as the ratio of migrated cells over control. In the presence of vandetanib $(5 \mu \mathrm{M})$ or AMD3100, the number of migrated cells for the two NB cell lines was significantly decreased compared with that of the control group $(\mathrm{p}<0.05)$. Furthermore, we found that the migrated cell number in the presence of vandetanib $(10 \mu \mathrm{M})$ was less than in the presence of AMD3100 and the difference was statistically significant $(\mathrm{p}<0.01)$. In addition, the combination of vandetanib and AMD3100 statistically increased its inhibitory effect on the migration of NB cells compared with the vandetanib-alone group $(\mathrm{p}<0.05)$ (Fig. 5). These data suggested that the migration of NB cells may be inhibited by vandetanib and the SDF-1/CXCR4 axis played an important role in NB migration; however, further studies need to be performed to determine whether vandetanib inhibits NB cell migration via the SDF-1/CXCR4 axis.

Vandetanib inhibits human NB cell invasion in vitro. Invasion is another important part of metastasis. To investigate the inhibitory effect of vandetanib on NB cell invasion, a Matrigel BioCoat invasion chamber with $8-\mu \mathrm{m}$ pores was used to assess the effect on cell invasion. We found that when treated with vandetanib, the number of both SK-N-SH and SH-SY5Y cells that travelled through the Matrigel BioCoat micropore membrane was significantly decreased compared with the control group $(\mathrm{p}<0.01)$ (Fig. 6) and we also found that AMD3100 blocked reduced cell invasion $(\mathrm{p}<0.05)$. The combination of vandetanib and AMD3100 statistically decreased cell invasion compared with the control group $(\mathrm{p}<0.01)$.

Vandetanib suppresses the expression of CXCR4 and MMP14 mRNA in human NB cell lines. RT-qPCR was performed to examine the expression of CXCR4, MMP14 and RET mRNA in NB cells treated with vandetanib. The results demonstrated that the CXCR4 and MMP14 mRNA expression levels in NB cell lines treated with vandetanib for $48 \mathrm{~h}$ were significantly lower than in cells treated with vehicle (DMSO) for $48 \mathrm{~h}$ $(\mathrm{p}<0.01)$ (Fig. 7), and there was no significant decrease in expression in cells treated with vandetanib for $72 \mathrm{~h}$ compared with those treated with vandetanib for $48 \mathrm{~h}$. There were no statistically significant differences in the RET mRNA expression levels among the treatment groups, although increasing expression of RET was observed as the concentration of vandetanib increased.

Vandetanib suppresses expression of the CXCR4 and MMP14 protein in human NB cell lines. The levels of both total expression and cell surface expression were examined by western blotting and immunofluorescence. Our results indicated that SK-N-SH and SH-SY5Y cells exposed to vandetanib for $48 \mathrm{~h}$ showed a significant decrease in total CXCR4 and MMP14 protein levels when compared with the vehicle control, and these levels remained suppressed with the extension of treatment time (Fig. 8A). In addition, immunofluorescence analysis indicated that phosphorylated RET and CXCR4 protein expression were decreased on the cell surface of SH-SY5Y cells treated with vandetanib for $48 \mathrm{~h}$ (Fig. 8B).

\section{Discussion}

Vandetanib is a novel small molecule inhibitor of the RET tyrosine kinases, VEGFR and EGFR with the potential to exert anticancer effects via multiple independent pathways. Vandetanib has demonstrated significant antitumor activity against a panel of diverse types of human cancer in clinical trials $(16,17)$. Our results showed that vandetanib may inhibit the proliferation of human NB cell lines, consistent with previous studies $(14,15)$. Our study also revealed an increased 

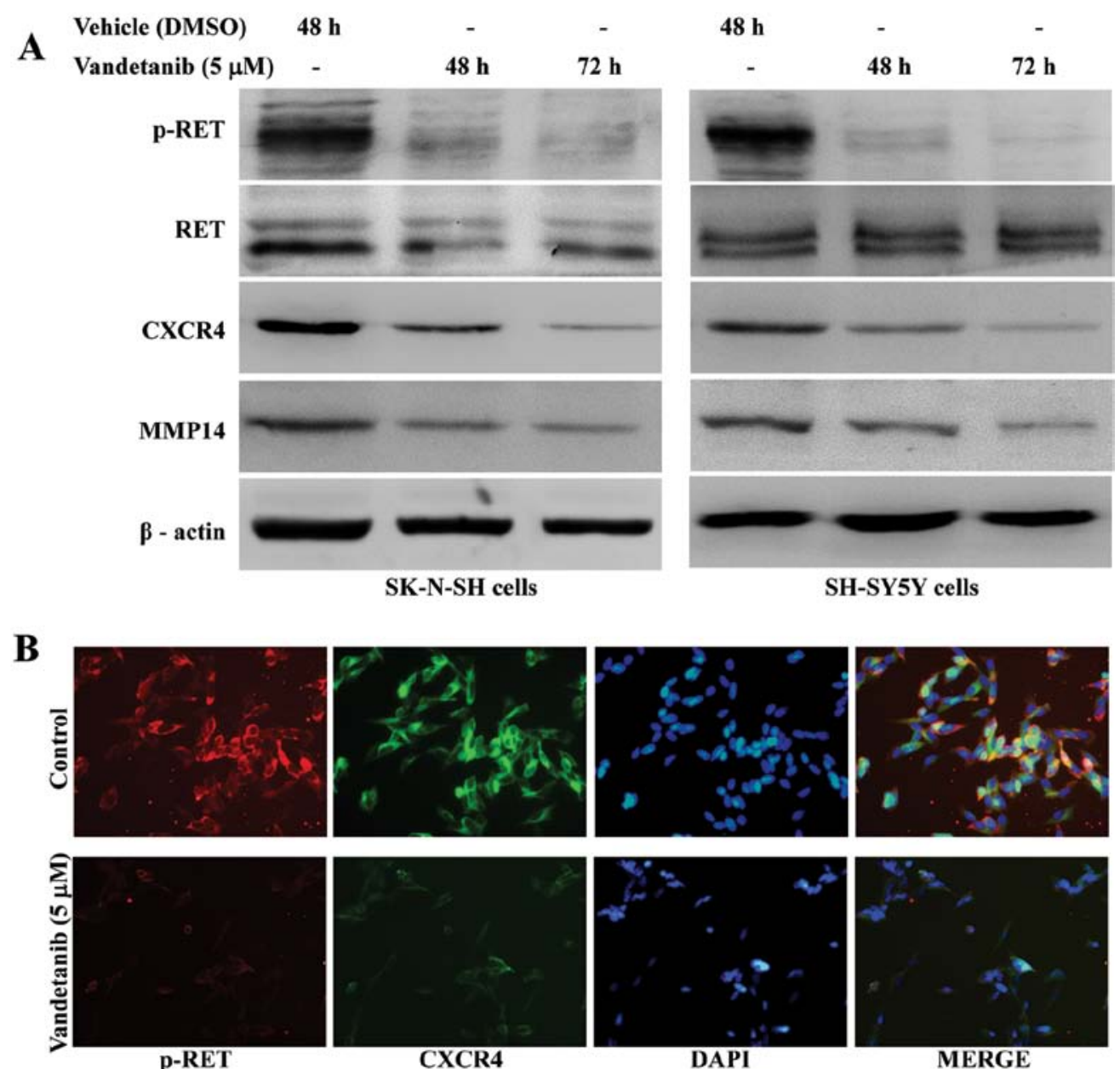

Figure 8. CXCR4 and MMP14 protein expression analysis in human neuroblastoma cell lines following DMSO and vandetanib treatment. (A) Western blot analysis of protein expression in human SK-N-SH and SH-SY5Y cells treated with DMSO and vandetanib, to evaluate CXCR4 and MMP14 expression. Similar results were obtained in three independent experiments in two neuroblastoma cells lines and a representative blot is shown. (B) Immunofluorescent images showed that vandetanib suppresses CXCR4 expression in SH-SY5Y cells. Cells were stained with TRITC (red)-conjugated anti-pRET and FITC (green)conjugated anti-CXCR4. All samples were counterstained with DAPI (blue). The experiment was performed in duplicate.

percentage of apoptotic cells and a markedly increased percentage of G1 phase cells in NB cell lines after treatment with $10 \mu \mathrm{M}$ vandetanib. The percentage of apoptotic cells increased significantly with increasing vandetanib concentrations up to $20 \mu \mathrm{M}$. These data suggested that vandetanib inhibits the proliferation of NB cells and that this inhibition is mediated by the induction of G1 phase cell cycle arrest at lower concentrations and apoptosis at higher concentrations.

Previous studies have shown that overexpression or activation of RET alters cell adhesion and enhances the metastatic behavior of NB $(3,4)$. Our current results showed vandetanib concentrations of $5 \mu \mathrm{M}$, which do not induce apoptosis, may inhibit the growth of human NB cell lines and RET phosphorylation in those cells. A randomized, double-blind phase III trial has demonstrated the significant antitumor activity of vandetanib in patients with locally advanced or metastatic medullary thyroid cancer, a challenging group in which all patients with hereditary MTC and approximately half of the patients with sporadic MTC suffered mutations in the RET proto-oncogene (18). In our study, vandetanib proved to be effective in inhibiting the migration of NB cells in vitro. Similar results were obtained in an invasion assay as the number of both SK-N-SH and SH-SY5Y cells treated with vandetanib that travelled through the Matrigel BioCoat micropore membrane was significantly decreased. These data suggest that vandetanib may be effective in the prevention of NB cancer cell migration and invasion in vitro. A previous study demonstrated that the migration of NB towards SDF-1 may be suppressed by AMD3100 or silencing of CXCR4, and also both CXCR4 silencing and AMD3100 inhibition reduced invasion (7). Our study showed that AMD3100 significantly suppressed the migration and invasion of NB cells, which is consistent with previous studies.

The CXCR4/SDF-1 axis has been well established as one of the most frequently expressed chemokines, which affect multiple pathways of tumor progression such as tumor cell proliferation, survival and metastasis in different types of tumors (19). In addition, a correlation between high CXCR4 expression in primary tumors and tumor metastasis was previously observed in NB patients $(20,21)$. Furthermore, another study demonstrated that functional expression of the CXCR4 chemokine receptor is induced by RET/PTC oncogenes in human papillary thyroid carcinomas, and CXCR4 induction depends on a specific tyrosine residue, tyrosine 1062, that is 
critical in mediating downstream signaling (22). In the present study, we showed that CXCR4 expression is inhibited by vandetanib in SK-N-SH and SH-SY5Y cells. This result was verified by qPCR, western blotting and immunofluorescence staining experiments. Based on the results of our study, we postulate that CXCR4 expression is regulated by RET/GDNF signaling in NB cells. It is noteworthy that migration in response to SDF- $1 \alpha$ and invasion of NB can be inhibited by both vandetanib and AMD3100, but it has been demonstrated that the inhibitory efficiency of vandetanib was stronger than that of AMD3100. Furthermore, the combination of vandetanib and AMD3100 statistically decreased cell migration and invasion compared with the vandetanib-alone group. Taken together, these observations support that vandetanib may inhibit the migration and invasion of NB and that this is associated with downregulation of the SDF-1/CXCR4 axis, but the axis is not enough to account for all the results observed.

Processes such as tumor growth, invasion and metastasis are strongly influenced by the surrounding microenvironment of the tumor, and altered extracellular proteolysis that changes these surroundings represents a major feature through which tumor cells are able to acquire specific functions necessary for tumor tissue invasion and metastasis. The matrix metalloproteinases (MMPs) represent the most prominent family of proteinases associated with tumorigenesis (23). In particular, matrix metalloproteinase 14 (MMP14), a well-known membrane type 1-matrix metalloproteinase (MT1-MMP), is closely associated with these processes. Mounting evidence supports a dominant role for MMP14 in the migration and invasion of metastatic tumor cells $(24,25)$. A report showed that microRNA-9 targets MMP14 to inhibit invasion, metastasis and angiogenesis of NB cells, which were rescued by overexpression of MMP14 (26). An early study demonstrated that MMP14 plays an important role in NB progression and that its expression is preferentially observed in tumor specimens from NB patients showing poor clinical outcomes (27). In light of previous findings in which the expression of MMP14 was upregulated by RET/GDNF signaling (6), we hypothesized that vandetanib may inhibit the expression of MMP14 through its inhibition of the RET tyrosine kinases. In the present study, we found that the expression of MMP14 mRNA and protein was significantly decreased in NB cells treated with vandetanib as compared to the control group. These data suggest that MMP14 also plays an important role in the inhibition of NB migration and invasion by vandetanib.

In conclusion, vandetanib may inhibit the proliferation of NB cells mediated by the induction of G1 phase cell cycle arrest and apoptosis and vandetanib may suppress the migration and invasion of NB cell lines in vitro. Therefore, vandetanib should be effective in the prevention of NB cancer cell migration and invasion. Furthermore, our data suggest that the potential mechanisms of vandetanib against migration and invasion of NB are partly attributed to suppression of the expression of CXCR4 and MMP14 in human NB cells by vandetanib. Collectively, our observations suggest that vandetanib may be developed as a new therapeutic strategy to control tumor growth and prevent NB metastasis to reduce the progression of relapse and refractoriness in NB patients. However, further animal experiments and clinical trials must be performed to determine its precise role in the treatment of NB metastasis.

\section{Acknowledgements}

The authors acknowledge the technical assistance from Mrs Qing Luo and Mr Xin Li. We appreciate the financial support provided by the National Natural Science Foundations of China (30973136).

\section{References}

1. Matthay KK, Reynolds CP, Seeger RC, et al: Long-term results for children with high-risk neuroblastoma treated on a randomized trial of myeloablative therapy followed by 13-cis-retinoic acid: a children's oncology group study. J Clin Oncol 27: 1007-1013, 2009.

2. Zage PE, Kletzel M, Murray K, et al: Outcomes of the POG 9340/9341/9342 trials for children with high-risk neuroblastoma: a report from the Children's Oncology Group. Pediatr Blood Cancer 51: 747-753, 2008.

3. Marshall GM, Peaston AE, Hocker JE, et al: Expression of multiple endocrine neoplasia 2B RET in neuroblastoma cells alters cell adhesion in vitro, enhances metastatic behavior in vivo, and activates Jun kinase. Cancer Res 57: 5399-5405, 1997.

4. Futami H and Sakai R: RET protein promotes non-adherent growth of NB-39-nu neuroblastoma cell line. Cancer Sci 100: 1034-1039, 2009.

5. Arighi E, Borrello MG and Sariola H: RET tyrosine kinase signaling in development and cancer. Cytokine Growth Factor Rev 16: 441-467, 2005.

6. Lu BC, Cebrian C, Chi X, et al: Etv4 and Etv5 are required downstream of GDNF and Ret for kidney branching morphogenesis. Nat Genet 41: 1295-1302, 2009.

7. Ma M, Ye JY, Deng R, Dee CM and Chan GC: Mesenchymal stromal cells may enhance metastasis of neuroblastoma via SDF-1/CXCR4 and SDF-1/CXCR7 signaling. Cancer Lett 312: $1-10,2011$.

8. Uygur B and Wu WS: SLUG promotes prostate cancer cell migration and invasion via CXCR4/CXCL12 axis. Mol Cancer 10: 139, 2011.

9. Wendel C, Hemping-Bovenkerk A, Krasnyanska J, et al: CXCR4/CXCL12 participate in extravasation of metastasizing breast cancer cells within the liver in a rat model. PLoS One 7: e30046, 2012.

10. Bialek J, Kunanuvat U, Hombach-Klonisch S, et al: Relaxin enhances the collagenolytic activity and in vitro invasiveness by upregulating matrix metalloproteinases in human thyroid carcinoma cells. Mol Cancer Res 9: 673-687, 2011.

11. Johnson JL, Pillai S, Pernazza D, Sebti SM, Lawrence NJ and Chellappan SP: Regulation of matrix metalloproteinase genes by E2F transcription factors: Rb-Raf-1 interaction as a novel target for metastatic disease. Cancer Res 72: 516-526, 2012.

12. Carlomagno F, Vitagliano D, Guida T, et al: ZD6474, an orally available inhibitor of KDR tyrosine kinase activity, efficiently blocks oncogenic RET kinases. Cancer Res 62: 7284-7290, 2002.

13. Wedge SR, Ogilvie DJ, Dukes M, et al: ZD6474 inhibits vascular endothelial growth factor signaling, angiogenesis, and tumor growth following oral administration. Cancer Res 62: 4645-4655, 2002.

14. Beaudry P, Nilsson M, Rioth M, et al: Potent antitumor effects of ZD6474 on neuroblastoma via dual targeting of tumor cells and tumor endothelium. Mol Cancer Ther 7: 418-424, 2008.

15. Zage PE, Zeng L, Palla S, et al: A novel therapeutic combination for neuroblastoma: the vascular endothelial growth factor receptor/epidermal growth factor receptor/rearranged during transfection inhibitor vandetanib with 13-cis-retinoic acid. Cancer 116: 2465-2475, 2010.

16. Herbst RS, Sun Y, Eberhardt WE, et al: Vandetanib plus docetaxel versus docetaxel as second-line treatment for patients with advanced non-small-cell lung cancer (ZODIAC): a double-blind, randomised, phase 3 trial. Lancet Oncol 11: 619-626, 2010.

17. Meyerhardt JA, Ancukiewicz M, Abrams TA, et al: Phase I study of cetuximab, irinotecan, and vandetanib (ZD6474) as therapy for patients with previously treated metastastic colorectal cancer. PLoS One 7: e38231, 2012.

18. Wells SA Jr, Robinson BG, Gagel RF, et al: Vandetanib in patients with locally advanced or metastatic medullary thyroid cancer: a randomized, double-blind phase III trial. J Clin Oncol 30: 134-141, 2012. 
19. Mantovani A, Savino B, Locati M, Zammataro L, Allavena P and Bonecchi R: The chemokine system in cancer biology and therapy. Cytokine Growth Factor Rev 21: 27-39, 2010.

20. Zhang L, Yeger H, Das B, Irwin MS and Baruchel S: Tissue microenvironment modulates CXCR4 expression and tumor metastasis in neuroblastoma. Neoplasia 9: 36-46, 2007.

21. Russell HV, Hicks J, Okcu MF and Nuchtern JG: CXCR4 expression in neuroblastoma primary tumors is associated with clinical presentation of bone and bone marrow metastases. J Pediatr Surg 39: 1506-1511, 2004.

22. Castellone MD, Guarino V, De Falco V, et al: Functional expression of the CXCR4 chemokine receptor is induced by RET/PTC oncogenes and is a common event in human papillary thyroid carcinomas. Oncogene 23: 5958-5967, 2004.

23. Kessenbrock K, Plaks V and Werb Z: Matrix metalloproteinases: regulators of the tumor microenvironment. Cell 141: 52-67, 2010 .
24. Zarrabi K, Dufour A, Li J, et al: Inhibition of matrix metalloproteinase 14 (MMP-14)-mediated cancer cell migration. J Biol Chem 286: 33167-33177, 2011.

25. Perentes JY, Kirkpatrick ND, Nagano S, et al: Cancer cell-associated MT1-MMP promotes blood vessel invasion and distant metastasis in triple-negative mammary tumors. Cancer Res 71: 4527-4538, 2011.

26. Zhang H, Qi M, Li S, et al: microRNA-9 targets matrix metalloproteinase 14 to inhibit invasion, metastasis, and angiogenesis of neuroblastoma cells. Mol Cancer Ther 11: 1454-1466, 2012.

27. Nyalendo C, Sartelet H, Barrette S, Ohta S, Gingras D and Béliveau R: Identification of membrane-type 1 matrix metalloproteinase tyrosine phosphorylation in association with neuroblastoma progression. BMC Cancer 9: 422, 2009. 